\title{
Implementation of ACA and Health Insurance Exchanges: The Challenges Ahead
}

\section{David Randall*}

David Randall, American Research and Policy Institute, Suite 200 1250, Connecticut Ave, NW, Washington, DC, USA

The Patient Protection and Affordable Care Act of 2010 (ACA) allows for the creation of state-based health insurance exchanges (HIX) and relies on states to make health insurance readily available to qualified population segments by 2014 [1]. A HIX is one mechanism for organizing the health insurance marketplace to help consumers and small businesses shop for coverage that allows for easy price comparisons [2]. The stated goal of the exchange mechanism is to create a more efficient, competitive market place for consumers and small employers. According the U.S. Department of Health and Human Services (HHS) this goal is accomplished by helping individuals shop, select, and enroll in high quality, affordable private health insurance products. In addition, the state-based HIX will also assist eligible individuals with premium tax credits, enrollment in eligible federal or state health care programs (such as Medicaid) and provide one-stop shopping for health insurance products.

In the "Initial Guidance to States on Exchanges," HHS establishes key principles and priorities for states in the establishment and certification of the HIX by January 1, 2013. The guidance offered by HHS can best summarized as follows:

- $\quad$ Promoting Efficiency

- $\quad$ Avoiding Adverse Selections

- Access and Continuity of Care

- Public Accountability and Transparency

- Financial Accountability

Each of the above general principles are important to delineate and expand when assessing the policy choices and ultimate HIX administrative and data architecture a state must employ to be certified by HHS and subsequent implementation [3]. As states and the federal government continue to modify the system architecture of the exchange websites, there are several issues worth exploring as potential implementation hurdles for successful operation of the exchanges after health insurance policies are issued and take effect in 2014.

One of the primary technical challenges of developing a data exchange is the integration of disparate data sources. This challenge is particularly important if an exchange is to be compliant with ACA statue because of different government agency data that needs to be linked. One of the most basic challenges is the linkage and posting of premium and payment information to an exchange. Additional integration of data from underwriting analysis to update premiums and permit a consumer to close a transaction must also be permitted.

A major developmental concern is whether an exchange will use a batch process or real time data for operations. Integrating real time data feeds across platforms can be a challenge for any data organization. One major consideration is whether the data exchange will be asynchronous (one-way) or synchronous (two-way) from different data stores. A classic example of an asynchronous data exchange would be the transmission of a web-based form of the entire beneficiary's available information from a broker to an underwriter. This is currently the practice of many e-insurance web sites that simply automate the broker and underwriter data intake [4].
A more complicated synchronous exchange is where the underwriter of an insurer can respond automatically based on quick review of the web-based form with premium quote. An alternative to real-time processing could be batch processing where routinely summarized data is integrated into the insurance purchase decision. For example, batch processed data would be a set of premium and benefit combinations from a set of insurers that are updated on a weekly basis. Additional eligibility and verification data for public insurance program could be made available electronically every two weeks. The main disadvantages of batch processed information are the potential for fraud and the lack of consumer engagement. The major advantages of real time information are fraud suppression through real-time verification of identity and better customer capture. Equally important is the verification and potential reconciliation of subsidies that are applied prospectively.

Consumers may be eligible for subsidies in the form of tax credits to help pay for their health care premium costs under the ACA. Premium tax credits would be refundable and advanceable. A refundable tax credit is one that is available to a person even if he or she has no tax liability. An advanceable tax credit (prospectively applied) allows a person to receive assistance at the time that they purchase insurance in the HIX rather than paying their premium out of pocket and waiting to be reimbursed when filing their annual income tax return [5].

In a real time inquiry to one or more federal databases, a prospective enrollee will be given a subsidy eligibility dollar amount that they may use toward their purchase of health care benefits. A consumer is not required to use their entire subsidy eligibility amount and may subjectively select a lower amount depending on their known income and tax status. This choice of elected subsidy amount as a subset of eligible amount adds complexity in reconciling "actual" subsidies used between the participating health plans, the HIX and the U.S. Department of the Treasury (Treasury).

The ACA subsidy payment workflow has the Treasury paying each Qualified Health Plan (QHP) directly instead of paying the all subsidies through the centralized accounting functions at the state HIX or Federally Facilitated Marketplace (FFM). This means that the Treasury must trust each QHP to take in and apply all current period subsidies, adjustments for prior periods, and new subsidy requests equally well.

*Corresponding author: David Randall, American Research and Policy Institute, Suite 200 1250, Connecticut Ave, NW, Washington, DC 20036, USA, Tel: (202) 558-2304 118; Fax: (202) 446-2964; E-mail: info@arapi.org

Received November 26, 2013; Accepted November 27, 2013; Published November 29, 2013

Citation: Randall D (2013) Implementation of ACA and Health Insurance Exchanges: The Challenges Ahead. Health Care Current Reviews 1: e101. doi: 10.4172/2375-4273.1000e101

Copyright: (c) 2013 Randall D. This is an open-access article distributed under the terms of the Creative Commons Attribution License, which permits unrestricted use, distribution, and reproduction in any medium, provided the original author and source are credited. 
Citation: Randall D (2013) Implementation of ACA and Health Insurance Exchanges: The Challenges Ahead. Health Care Current Reviews 1: e101. doi: 10.4172/2375-4273.1000e101

Page 2 of 2

It also assumes the Treasury itself will provide accurate centralized accounting for all subsidies paid through the Federal and State HIX necessary to know when the Treasury expects reimbursements for over-payments, and refunds resulting from termination of an enrollee for non-payment of premium. Grace Period, delinquency and termination rules will create delayed payments to health plans, data discrepancies and significant volumes of retroactivity between the QHPs, the HIX and Treasury. The churn of low income enrollees between QHPs and Medicaid will introduce further complexity by creating multiple discrete periods of subsidy eligibility within a single plan year. Policymakers and practitioners should be aware of these potential implementation hurdles beyond the current enrollment issues associated with initial eligibility and enrollment determination, especially given the complexities associated with subsidy calculations and reconciliation.

\section{References}

1. A Health Insurance Exchange is defined under Section 1311(b) of the Patien Protection and Affordable Care Act of 2010.(Public Law 111-148), as amended by the Healthcare and Reconciliation Act of 2010 (Public 111-152).

2. Center for Consumer Information and Insurance Oversight "Initial Guidance to States on Exchanges"

3. States are evaluated by a minimum 11 core elements to be certified by the January 1, 2013 deadline.

4. www.ehealthinsurance.com

5. www.kff.org/healthreform/8061.cfm 